\title{
USING VERY FAST SIMULATED ANNEALING IN THE WEIGHTED AB SEMBLANCE METHOD
}

\author{
Marcelo Santana de Souza ${ }^{1}$ and Milton José Porsani ${ }^{2}$
}

\begin{abstract}
The conventional velocity analysis does not consider AVO effects in reflection seismic data. These conditions lead to obtaining of inadequate velocity fields, making it difficult to execute other steps in seismic processing. To overcome this problem, researchers developed the Weighted AB semblance method, a coherence measure which deals with AVO effects in velocity spectra. It is based on the application of two sigmoid weighting functions to AB semblance, which depend on four coefficients. The values of these coefficients directly influence the resolution of the resulting velocity spectrum. In this work, we apply the inversion algorithm Very Fast Simulated Annealing (VFSA) to obtain these values. Numerical experiments show that VFSA is a quite effective method, obtaining correct coefficient values and allowing the generation of the velocity spectrum with an excellent resolution for both synthetic and real data. Results also proved that Weighted AB semblance is an optimal coherence measure to be used in velocity spectrum, because it is insensitive to AVO effects and reversal polarity and presents considerably a better resolution than conventional semblance.
\end{abstract}

Keywords: velocity analysis, AV0, high-resolution velocity spectra.

RESUMO. A análise de velocidades convencional não considera efeitos de AVO em dados sísmicos de reflexão. Essas condições levam à obtenção de campos de velocidades inadequados, dificultando a execução de outras etapas do processamento sísmico. Para superar esse problema, pesquisadores desenvolveram o método AB semblance Ponderado, uma medida de coerência que lida com efeitos de AVO em espectros de velocidades. Ela é baseada na aplicação de duas funções sigmoides à $A B$ semblance, que depende de quatro coeficientes. Os valores desses coeficientes influenciam diretamente a resolução do espectro de velocidade resultante. Nesse trabalho, nós aplicamos o algoritmo de inversão Very Fast Simulated Annealing (VFSA) para obter esses valores. Experimentos numéricos mostram que VFSA é um método bastante eficaz, obtendo valores corretos dos coeficientes e permitindo a geração do espectro de velocidade com uma excelente resolução tanto para dados sintéticos quanto para dados reais. Resultados também provam que o AB semblance Ponderado é uma medida de coerência ótima para ser usada no espectro de velocidade, porque ela é insensível aos efeitos de AVO e apresenta resolução consideravelmente melhor do que a semblance convencional.

Palavras-chave: análise de velocidades, AVO, espectro de velocidades de alta resolução.

Corresponding author: Marcelo Santana de Souza

${ }^{1}$ Universidade Federal da Bahia, Centro de Pesquisa em Geofísica e Geologia. Instituto de Geociências. Campus Universitário da Federação Salvador, BA, Brazil E-mail:marcelo.geofisica@gmail.com

2Universidade Federal da Bahia, Instituto de Geociências, Centro de Pesquisa em Geofísica e Geologia e Instituto Nacional de Ciência e Tecnologia de Geofísica do Petróleo - INCT-GP. Campus Universitário da Federação Salvador, BA, Brazil -E-mail: porsani@ufba.br 


\section{INTRODUCTION}

Velocity analysis is an essential step in the CMP seismic processing, since the resulting velocity field directly affects the quality of the image of the Earth's interior. Velocity analysis methods aim to estimate the wave velocity in subsurface from measurements done on surface. The most conventional way of obtaining the velocity field is using the velocity spectrum, based on a coherence measure to determine a stacking velocity function of time that characterizes all events. This method is suitable for media with small lateral variation of velocity and it is still quite useful for building the initial velocity model.

In conventional velocity analysis, the effects of AVO (Amplitude Variation with Offset) are usually discarded. This assumption is quite practical, but it does not describe what actually occurs in seismic data, and it is ineffective in interfaces associated with anomalies of AVO of classes 1 and 2 (Rutherford \& Williams, 1989). The semblance (Taner \& Koehler, 1969) is the most conventional coherence measure used in velocity spectra. It is considered robust for most practical situations, but it does not perform well in data with strong AVO effects, especially when reversal polarity occurs. To overcome these problems, many authors have attempted to develop other coherence measurements that are insensitive to AVO effects, and present a better resolution than conventional semblance. Sarkar et al. (2001) and Sarkar et al. (2002) have developed the AB semblance and AK semblance, respectively, which are insensitive to AVO effects and to the extreme case of reversal polarity. As studies progress, the so-called high-resolution velocity spectra have emerged (Biondi \& Kostov, 1989; Sacchi, 1998; Abbad \& Ursin, 2012). One of the kinds of these velocity spectra is that based on the modification of the eigenstructure of the data, as proposed by Biondi \& Kostov (1989), Barros (2012), Ursin et al. (2014). These methods are less sensitive to noise and present better resolution than conventional semblance. Other methods are based on the multiplication of weighting functions to the values of the conventional semblance or other coherence measure, such as $A B$ semblance. These functions are applied in order to increase or decrease the coherence values, depending on the quality of the NMO correction applied to the data Luo \& Hale (2012); Chen et al. (2015). Recently, Ebrahimi et al. (2017b) and Ebrahimi et al. (2017a) developed the Weighted AB semblance method, based on the multiplication of two sigmoid weighting functions to $\mathrm{AB}$ semblance. One of the functions, called $W_{S V D}$, is based on the ratio between the first and the second singular values of the considered moving window, while the other, $W_{P O W}$, is based on the position of the event within this window.

These functions depend on four coefficients that control the slope and the turning point of the curve. These coefficient estimates are obtained by analysis of a matrix that measures the sparsity of the spectrum, which is difficult to interpret. In this work, we present a new alternative to calculate the coefficients by means of the inversion method Very Fast Simulated Annealing (VFSA), which has many applications in Geophysics (Sen \& Stoffa, 1995; Soares, 2013; Santos \& Porsani, 2017). We performed tests in synthetic and real data. The numerical results show that the VFSA provided the correct coefficient values, generating velocity spectra that are insensitive to the reversal polarity and present resolution considerably better than conventional semblance, making it easier to determine stacking velocity functions.

\section{METHODOLOGY}

\section{The reversal polarity phenomenon}

Reflection seismic events in real data present Amplitude Variation with Offset (AVO). This effect is directly related to elastic properties of rocks in subsurface and is more anomalous in gas-sand reservoirs. The two factors that most determine the AVO behavior are the acoustic impedance and the Poisson's ratio contrasts at the reflectors (Ostrander, 1984). Rutherford \& Williams (1989) divided the AVO anomalies in three classes, using the impedance contrast with the encasing rock as the criterion. The classes are:

- Class 1: high-impedance sands;

- Class 2: near-zero impedance contrast sands;

- Class 3: low-impedance sands.

For the classes 1 and 2, AVO can cause reversal polarity in seismic traces. In these types of anomalies, the reflection coefficient is initially positive for the short-offset traces and decreases its value as incidence angle increases. The reversal polarity is a 180-degree change in the phase of a seismic reflection (Keys, 1989), and it occurs when P-wave reflection coefficients become negative as incidence angle varies. Its presence can cause amplitude cancellation in CMP stacking, making it difficult to interpret the stacked section. In velocity spectra, reversal polarity suppresses the amplitudes related to reflections. Figure 1 shows a synthetic CMP gather containing a reflection event that presents reversal polarity. Note that, initially 
the amplitudes are positive for short offsets and decrease their values for intermediate offsets until becomes negative for long offsets.

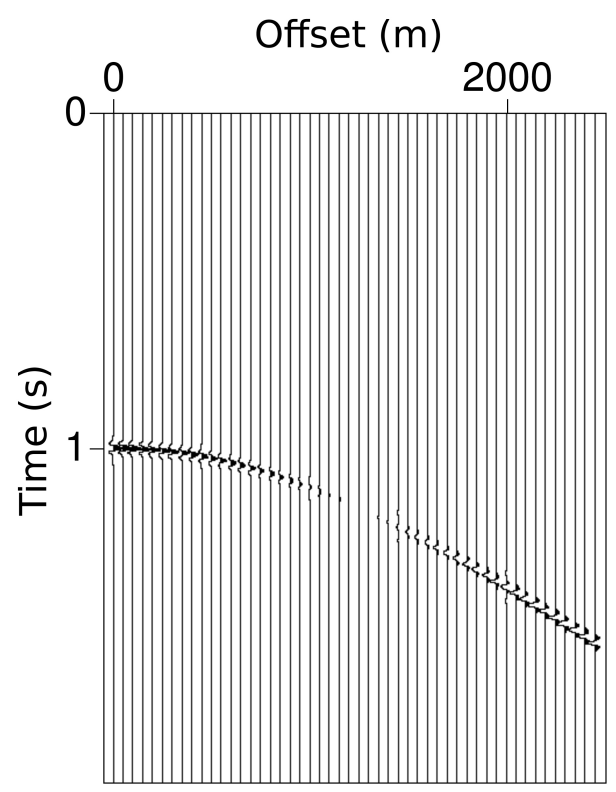

Figure 1-CMP gather containing one reflection event with reversal polarity.

\section{The velocity spectrum}

The velocity spectrum is a tool that allows analyzing the energy of reflections in the zero-offset time $\times$ stacking velocity domain. Its generation can be exemplified in Figure 2 as follows: Given a CMP gather containing five reflections with velocities equal to $1500 \mathrm{~m} / \mathrm{s}, 1750 \mathrm{~m} / \mathrm{s}, 2000 \mathrm{~m} / \mathrm{s}, 2250 \mathrm{~m} / \mathrm{s}$ and $2500 \mathrm{~m} / \mathrm{s}$ (Fig.3a), firstly, an interval of velocities is chosen, varying from a minimum value, $v_{\min }$, to a maximum value $v_{\max }$. Then, for each velocity, one corrects the CMP gather for NMO (Fig. 2b), arranging the data in a cube of dimension NS time samples $x$ $N X$ traces $\times N$ velocities. For each velocity, we have a slice of the cube that is the CMP gather corrected for NMO. To represent the reflection in velocity spectrum, one applies a coherence measure, generally the semblance. Given a NMO-corrected CMP gather and considering a moving time window of $L$ samples, the semblance $S$ is computed as:

$$
S=\frac{1}{N} \frac{\sum_{i=t_{0}-L / 2}^{t_{0}+L / 2}\left(\sum_{j=1}^{N X} a_{i j}\right)^{2}}{\sum_{i=t_{0}-L / 2}^{t_{0}+L / 2}\left(\sum_{j=1}^{N X} a_{i j}^{2}\right)} ;
$$

where $a_{i j}$ represents the amplitude in the ith time sample and jth trace. The calculated semblance value is attributed to the central sample of the window, associated to some $t_{0}$ in the
CMP. Then this window is moved, and a new semblance value is computed, it is associated to a new $t_{0}$, and so on. This procedure is repeated for all CMP gathers in the cube, generating the velocity spectrum. Figure $2 \mathrm{c}$ exemplifies the computation of the semblance values for the panel corrected for NMO using $v_{N M O}=2000 \mathrm{~m} / \mathrm{s}$. The maximum value of coherence is in $t_{0}=1.0 \mathrm{~s}$, because there is an event that is properly flattened with this velocity. Semblance values are normalized between zero and one, and they are directly related to the coherence of the events, so that the correctly flattened events will have values close to one, corresponding to the peaks in the spectrum. On the other hand, the incorrectly flattened events will have low values, close to zero. After generating the velocity spectrum, a manual determination of the stacking velocities is generally done, by picking the velocities associated to the energy of the primary reflections, obtaining a velocity function which allows the correct stacking of all reflections, as shown in Figure 2d.

Semblance assumes that the amplitudes of a given event remain constant with offsets, which does not actually occur. The picking of velocities becomes imprecise in data that suffer with important AVO responses, mainly in extreme cases, associated with reversal polarity (Sarkar et al., 2001, 2002; Yan \& Tsvankin, 2008). Figure 3 shows a CMP gather containing one hyperbolic event with $v_{N M O}=2000 \mathrm{~m} / \mathrm{s}$, presenting reversal polarity in (a). Its corresponding velocity spectrum is shown in Figure $3 \mathrm{~b}$. Note that the determination of the correct velocity is practically impossible using the semblance, because the reflection's energy has been suppressed.

\section{AB semblance}

For dealing with the undesirable AVO effect in velocity spectra, Sarkar et al. (2001) developed a new coherence measure, called $A B$ semblance. Fomel (2009) defined $A B$ semblance as the squared correlation of the amplitudes $a_{i j}$ with a trend. For a time sample $i$, the trend $b_{i j}$ is:

$$
b_{i j}=A_{i}+B_{i} x_{j},
$$

where $x_{j}$ is a vector which contains the offsets for each trace $j$. Thus, we have:

$$
S_{A B}=\frac{1}{N} \frac{\sum_{i=1}^{M}\left(\sum_{j=1}^{N} a_{i j} b_{i j}\right)^{2}}{\sum_{i=1}^{M}\left(\sum_{j=1}^{N} a_{i j}^{2} \sum_{j=1}^{N} b_{i j}^{2}\right)} .
$$

If we replace the trend $b_{i j}$ by a constant value $C$, we have the conventional semblance, which can be interpreted as the squared correlation between a constant and a matrix. 


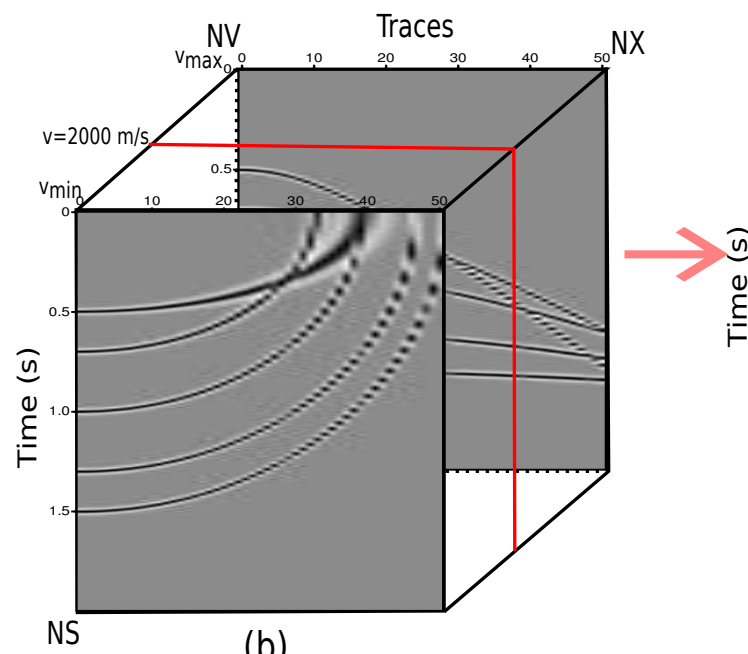

(b)

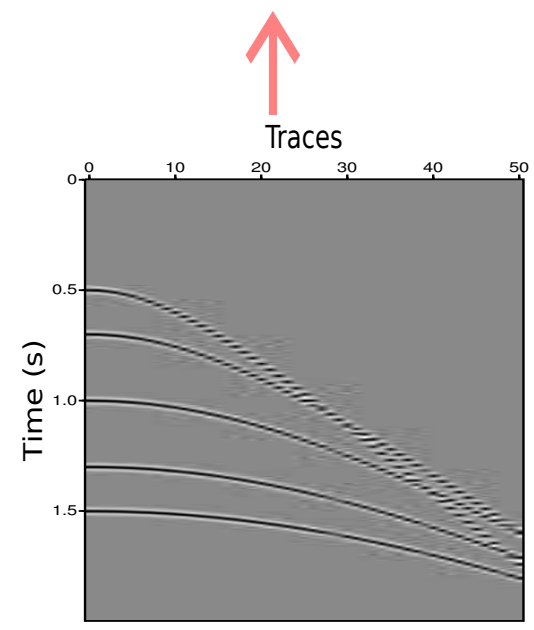

(a)

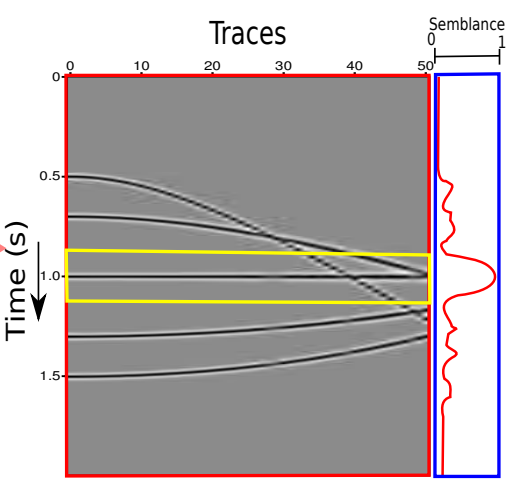

(c)

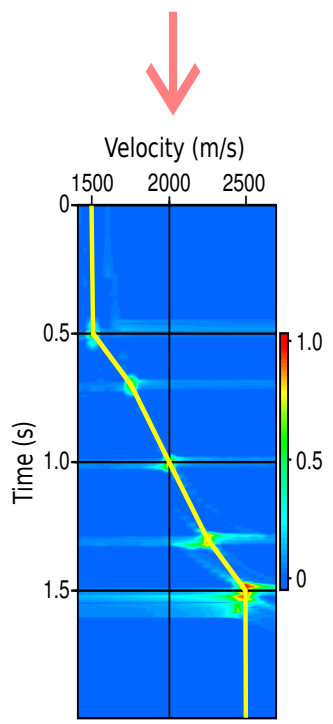

(d)

Figure 2 - Scheme of generation of the velocity spectrum for a synthetic CMP gather composed of five events: the CMP gather in (a), the cube containing NM0-corrected CMPs for each constant velocity in (b), the calculus of the semblance coherence measure for the NMO-corrected CMP with $v_{N M O}=2000 \mathrm{~m} / \mathrm{s}$ in (c), and the velocity spectrum and its respective velocity function (yellow line) in (d).

The values of $A$ and $B$ in Equation (2) can be found by minimization of the objective function:

$$
Q_{i}(A, B)=\sum_{j=1}^{N}\left(a_{i j}-A_{i}-B_{i} x_{j}\right)^{2} .
$$

Substituting $b_{i j}$ in Equation (3), utilizing $A_{i}$ and $B_{i}$ obtained by least-squares using Equation (4) we have the $\mathrm{AB}$ semblance coherence measure.

Fomel (2009) performed statistical analysis and numerical experiments, proving that $A B$ semblance is not significantly affected by AVO effects. On the other hand, it presents resolution that is approximately twice lower than conventional semblance, and it is twice more sensitive to noise than semblance.

\section{Weighted AB semblance}

Aiming to improve the semblance resolution, Ebrahimi et al. (2017b) developed a method based on the application of two weighting functions to the semblance values. Subsequently, this application was extended to the AB semblance (Ebrahimi et al., 2017a), aiming to generate a coherence measure which deals with AVO effects and presents a better resolution than conventional semblance. Weighted AB semblance is defined by:

$$
S_{w A B}=W_{S V D} \times W_{P O W} \times S_{A B}
$$

The sigmoid function $W_{S V D}$ is based on the ratio between the first and the second singular values, obtained by SVD 


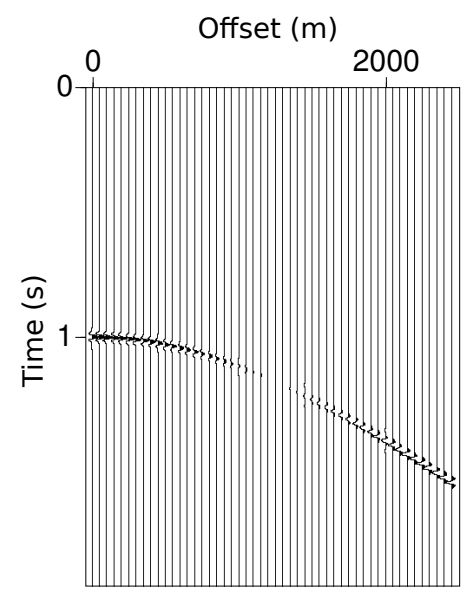

(a)

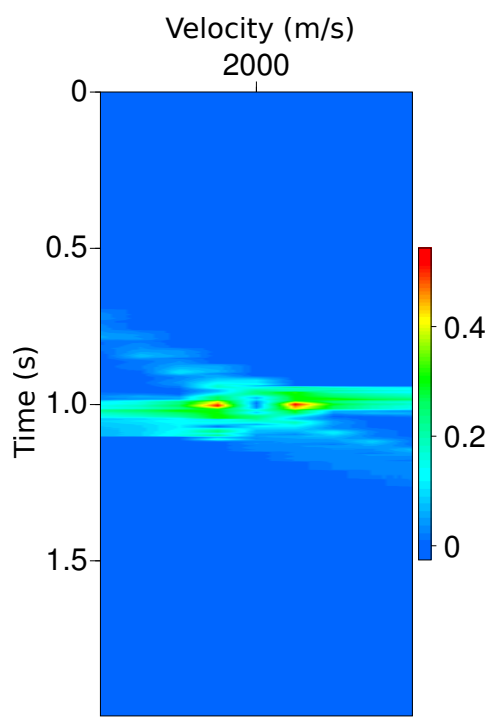

(b)

Figure 3 - Synthetic CMP gather containing one event with reversal polarity in (a) and its respective velocity spectrum using semblance as a coherence measure in (b).

decomposition applied to a moving time window. It is based on the property that, if a reflection event is properly NMO-corrected, it will present a high spacial correlation, and the first singular value $\sigma_{1}$ will be much greater than the second one $\sigma_{2}$. Thus, the $\sigma_{1} / \sigma_{2}$ ratio is a factor that makes $W_{S V D}$ very sensitive to variations in velocity, improving the spectrum's resolution in this direction.

$W_{S V D}$ is defined as:

$$
W_{S V D}=\frac{10}{1+e^{-a\left(\frac{\sigma_{1}}{\sigma_{2}}-b\right)}},
$$

where $a$ and $b$ are empirical constants. Thus, $W_{S V D}$ applies a higher weight when correct velocity values are used, enhancing these coherence values. On the other hand, if incorrect velocities are used, $W_{S V D}$ will have a small weight, so that the coherence values will be further decreased.

The sigmoid function $W_{P O W}$ is based on the position of the wavelet in the moving window. It is defined as:

$$
W_{\text {POW }}=\frac{100}{1+e^{-c(P O W-d)}},
$$

where $c$ and $d$ are empirical constants. POW (Position of Wavelet) is given by:

$$
P O W=\frac{1}{\left|t_{c m}-t_{\text {center }}\right|+\varepsilon},
$$

$t_{\text {center }}$ being the central time of the moving window, $\varepsilon$ is a small positive constant and $t_{c m}$ is the center of mass of the window, given by:

$$
t_{c m}=\frac{\sum_{i=1}^{M}\left(i \times \sum_{j=1}^{N}\left|a_{i j}\right|\right)}{\sum_{i=1}^{M} \sum_{j=1}^{N}\left|a_{i j}\right|} .
$$

According to Ebrahimi et al. (2017a), for a zero phase wavelet, the value of $\left|t_{c m}-t_{\text {center }}\right|$ in Equation (8) will be equal to zero if the central sample of the moving window coincides with the zero-offset time $t_{0}$ of the flattened event, considering correct values of velocity. Otherwise, if the central sample does not coincide with $t_{0}$, or an incorrect velocity is used, the denominator in Equation (8) will increase its value and, consequently, POW will decrease, so that a smaller weight will be applied by the $W_{P O W}$ function.

Figure 4 shows a comparison between values of the coherence measures semblance, $A B$ semblance and Weighted $A B$ semblance in function of the stacking velocity. For calculating these values was used a moving time window containing one event that presents reversal polarity and velocity of $1500 \mathrm{~m} / \mathrm{s}$, in which $t_{0}$ coincides with the central sample of the window. This event was flattened for various velocities. One realizes that conventional semblance suffers with the reversal polarity, presenting two peaks of coherence that are not associated with the correct velocity. However, $A B$ semblance and Weighted $A B$ 


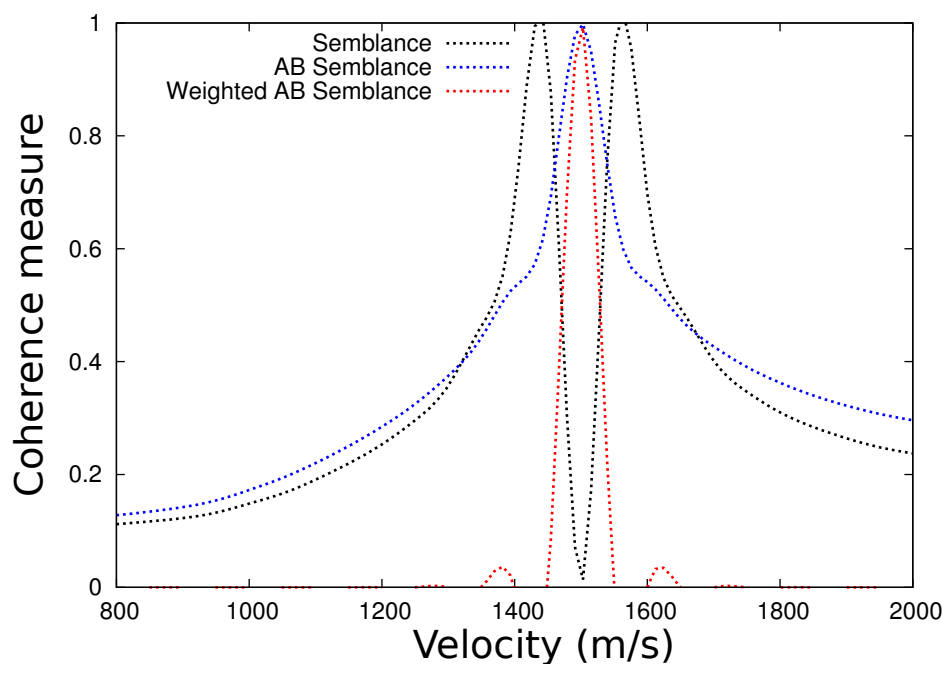

Figure 4 - Comparison between the coherence measures semblance, AB semblance and Weighted $A B$ semblance, calculated using a moving time window in which the event was flattened for various velocities.

semblance showed insensitive to the reversal polarity effect, but the latter presents a curve much more sharper.

Both pairs of coefficients in $W_{S V D}$ and $W_{P O W}$ functions control the slope and turning point, more precisely, by varying the values of $a$ or $c$, there is a change in the slope of the curve representing the function, whereas by varying the values de $b$ or $d$ moves the turning point. According to Ebrahimi et al. (2017a), values of the coefficient are related to the sparsity of the velocity spectrum. The coefficients are obtained by means of an analysis of a matrix which contains the measure $E C M$ (Energy Concentration of a Matrix). This measure is calculated by:

$$
E C M=\frac{1}{\sum_{i} \sum_{j}\left|x_{i j}\right|^{0,01}},
$$

where $x_{i j}$, in this case, are the amplitudes of the velocity spectra. The ECM matrix is generated as follows:

- Fix the coefficient values of one of the weighting functions;

- Generate velocity spectra using various values of the two other parameters;

- Calculate the ECM of the velocity spectra for each pair of coefficient values.

The higher the ECM value, the greater the sparsity of the velocity spectrum. Figure 5 shows ECM matrices for determination of the pairs $a$ and $b, c$ and $d$ for a synthetic CMP gather containing only one reflection with reversal polarity. The coefficient values are empirically determined. The analysis of these matrices consists in the attempt to select pairs that result in a velocity spectrum with optimal resolution. Once these pairs are selected, evaluates the resulting velocity spectrum and, if a good resolution is obtained, the pairs are chosen as optimal, otherwise, select new pairs, until obtains a velocity spectrum with optimal resolution. This step can be laborious, firstly due to the computational effort of, for example, $n a \times n b$ velocity spectra for the generation of the ECM matrix, being $n a$ and $n b$ the number of trial values of the coefficients $a$ and $b$, secondly, it is difficult to interpret the ECM matrix for obtaining the correct coefficients values.

\section{Very Fast Simulated Annealing}

The Very Fast Simulated Annealing (VFSA), first implemented by Ingber (1989), is a stochastic method of inversion based on modifications of the Simulated Annealing (SA) and Fast Simulated Annealing (FSA) methods. The main idea of these methods comes from thermodynamics, because they aim to simulate the cooling of a physical system, and to analyze the global state of its minimum energy (Sen \& Stoffa, 1995). One advantage of this method is its global scoping character which aims to avoid to get trapped in local minima of the objetive function to be optimized.

The VFSA method is described below (Sen \& Stoffa, 1995):

Assume that the model parameter $m^{i}$ at iteration $k$ is represented by $m_{k}^{i}$ 


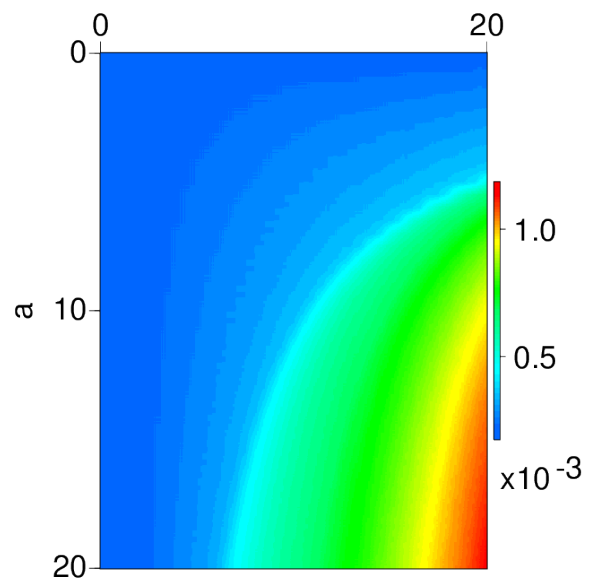

(a)

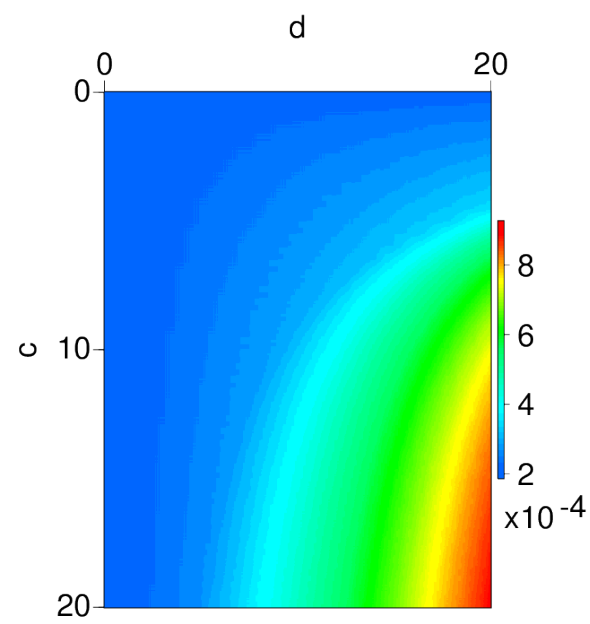

(b)

Figure 5 - ECM matrices for analysis of pairs of coefficients $a$ and $b$ in (a), and for analysis of pairs of coefficients $c$ and $d$ in (b).

$$
m_{\min }^{i} \leq m_{k}^{i} \leq m_{\max }^{i}
$$

where $m_{\min }^{i}$ and $m_{\max }^{i}$ are the minimum and maximum values of the model parameter $m_{k}^{i}$. The value of this parameter is perturbed in the iteration $k+1$ by using the following relation

$$
m_{k+1}^{i}=m_{k}^{i}+y^{i}\left(m_{\max }^{i}-m_{\min }^{i}\right),
$$

being $y^{i} \in[-1,1]$, generated from the following distribution

$$
g_{T}(y)=\prod_{i=1}^{N M} \frac{1}{2\left(\left|y_{i}\right|+T_{i}\right) \ln \left(1+\frac{1}{t_{i}}\right)}=\prod_{i=1}^{N M} g_{T i}\left(y_{i}\right) .
$$

Thus, a random number $u^{i}$, drawn from a uniform distribution $U[0,1]$ can be mapped into the above distribution with the following formula

$$
y_{i}=\operatorname{sgn}\left(u_{i}-\frac{1}{2}\right) T_{i}\left[\left(1+\frac{1}{T_{i}}\right)^{\left|2 u_{i}-1\right|}-1\right]
$$

For this distribution, we can statistically search for the global minimum by using the following cooling schedule:

$$
T^{i}(k)=T^{0 i} \exp \left(-c^{i} k^{1 / N M}\right)
$$

where $N M$ is the number of models, $T^{0 i}$ is the value of the initial temperature for the $i$ parameter, $c^{i}$ is an attribute used to control the temperature schedule. The criterion of acceptance of models is given by Metropolis algorithm (Metropolis et al., 1953) as follows:
- Start with an initial model $m_{0}$ with energy $E\left(m_{0}\right)$;

- Make a small pertubation to the model $m^{j}=m^{i}+\Delta m^{i}$;

- Calculate $\Delta E^{i j}=E\left(m^{j}\right)-E\left(m^{i}\right)$;

- If $\Delta E^{i j} \leq 0$, the new model is always accepted;

- If $\Delta E^{i j}>0$, the new model is accepted with the probability $P=\exp \left(\frac{\Delta E^{i j}}{T}\right)$.

\section{Obtaining the coefficients using VFSA}

In applying the VFSA method to determine the pairs of coefficients that result in a velocity spectrum with better resolution, the vector $\mathbf{m}$ of the model parameters will be composed of the coefficients used in the $W_{S V D}$ and $W_{P O W}$ functions, so we have $\mathbf{m}=(a, b, c, d)$. The $\mathrm{ECM}(\mathbf{m})$ measure will be equivalent to the objective function. Since the ECM is a measure whose value is directly proportional to the sparsity of a matrix, and the better the resolution the sparser it becomes, the velocity spectrum is proportional to the ECM value. Thus, the VFSA algorithm is applied to converge to a maximum, providing a set of coefficient values that will result in values close to the maximum ECM. Adapting the VFSA algorithm to obtain the optimal coefficients for generating the spectrum, the pseudo-code of the algorithm is: 


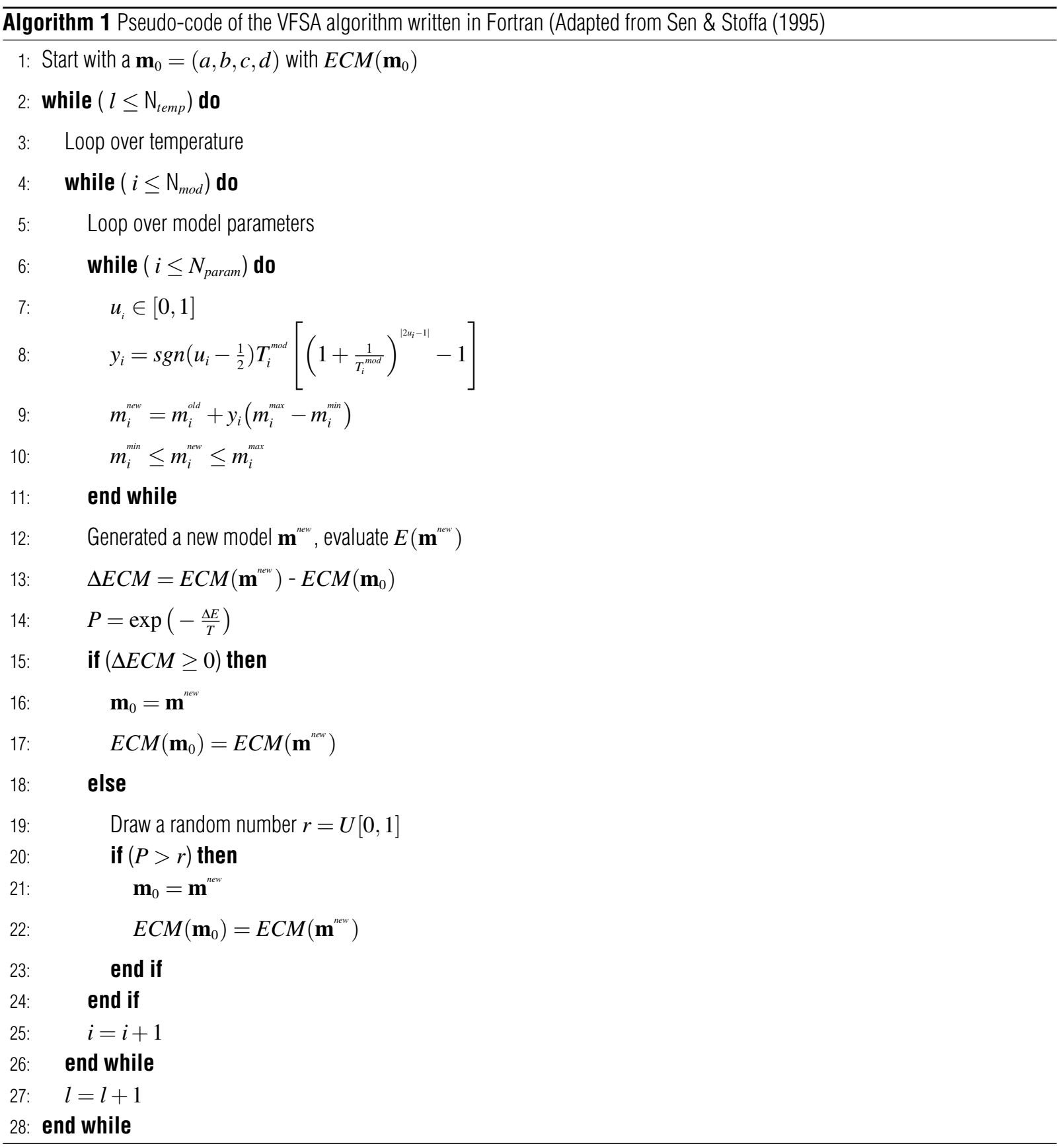

\section{RESULTS}

\section{Synthetic data}

We applied the VFSA method to a synthetic CMP gather with 50 traces, 1000 time samples, sampling interval of 4 milliseconds, containing one reflection event with $t_{0}=2.0 \mathrm{~s}$ and velocity of $1500 \mathrm{~m} / \mathrm{s}$. The event presents reversal polarity. In applying VFSA, we used 60 temperatures and 40 models, obtaining the coefficients $a=2.8, b=7.5, c=3.0$ and $d=2$.8. Figure 6 shows the synthetic CMP gather in (a) and its velocity spectra, normalized between zero and one (from Fig. 6b to Fig. 6d). The length of the moving window used in all velocity spectra was 20 ms. Comparing results, we conclude that conventional semblance suffered with the effect of reversal polarity. On the other hand, $A B$ semblance was not affected by this effect, but its resolution is poorer than conventional semblance. Weighted AB semblance overcame the AVO phenomenon and solved the problem of the 


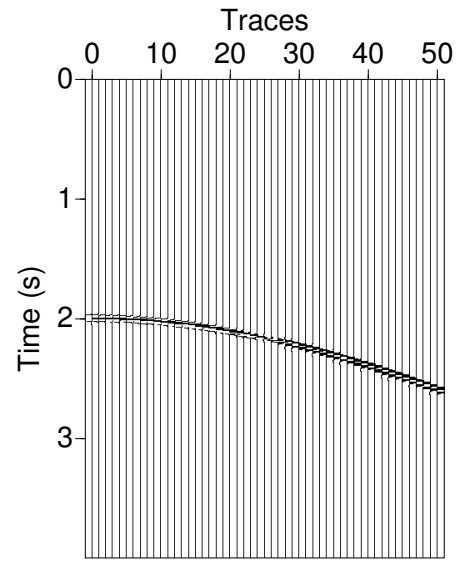

(a)

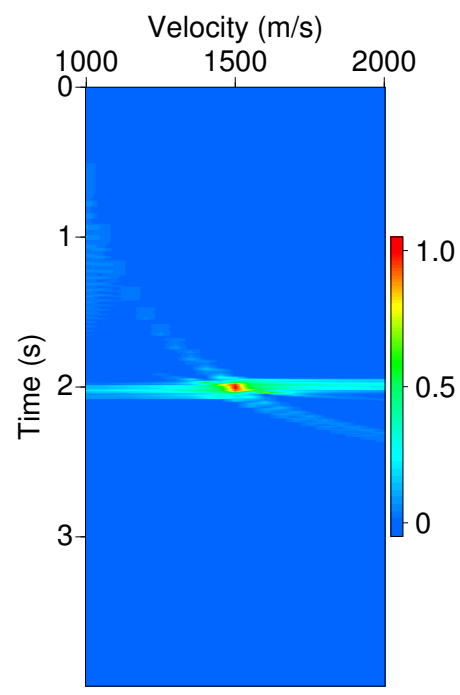

(c)

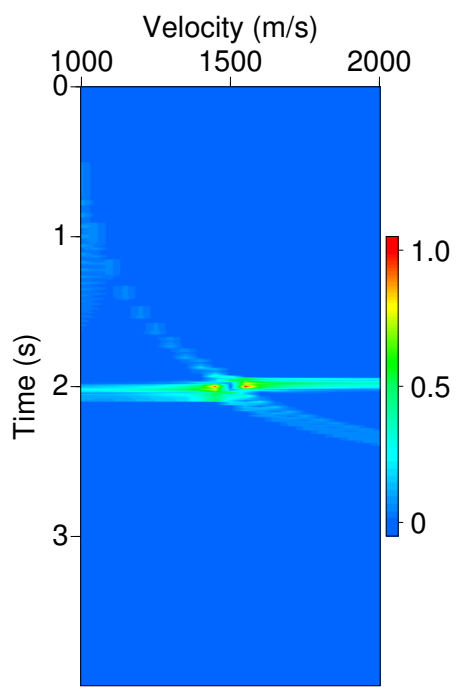

(b)

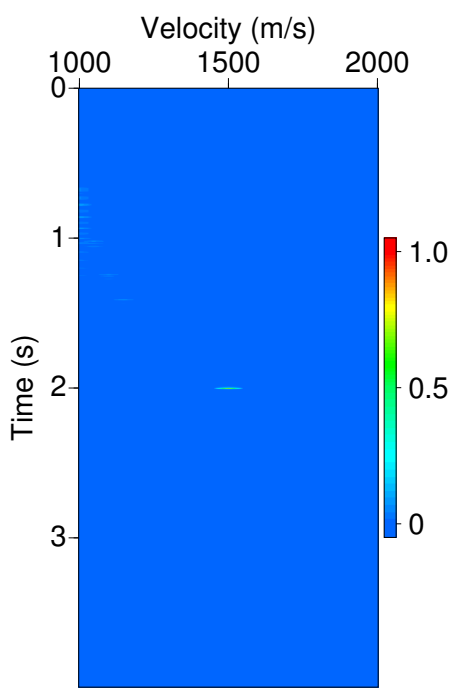

(d)

Figure 6 - Synthetic CMP gather in (a) and its velocity spectra using conventional semblance in (b), AB semblance in (c), and Weighted AB semblance in (d).

low-resolution of $A B$ semblance, validating the effectiveness on the use of the VFSA in the obtaining of the coefficients values.

To evaluate the effectiveness of the method in noisy data, we added $80 \%$ of random noise to the synthetic CMP containing one event as it is shown in Figure 7 . We notice that semblance suffered with the noise, losing its resolution even more, but the $A B$ semblance was much more affected by the noise. On the other hand, the Weighted $A B$ semblance was not affected by noise.

\section{Real data}

For real data, we tested the method in a marine real CMP gather from Jequitinhonha Basin, located in the southeast coast of Brazil. The data parameters are 1001 time samples, 60 traces and sampling interval of $4 \mathrm{~ms}$. These data do not present a strong AVO effect. Figure 8 shows the CMP gather in (a), and its respective velocity spectra using semblance, $A B$ semblance and Weighted $A B$ semblance in Figure 8b to Figure 8d, respectively. The length 


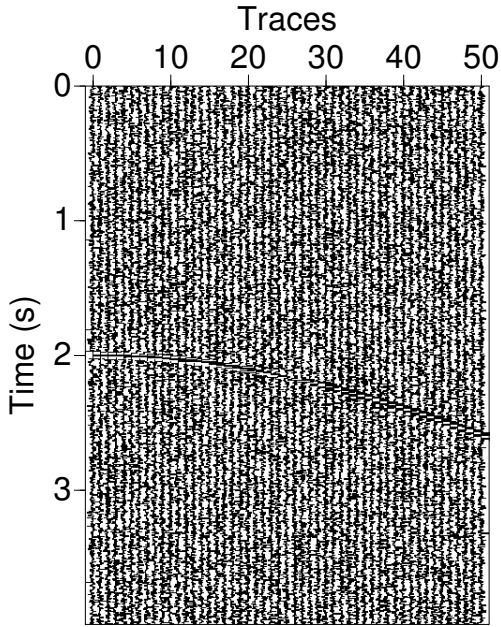

(a)

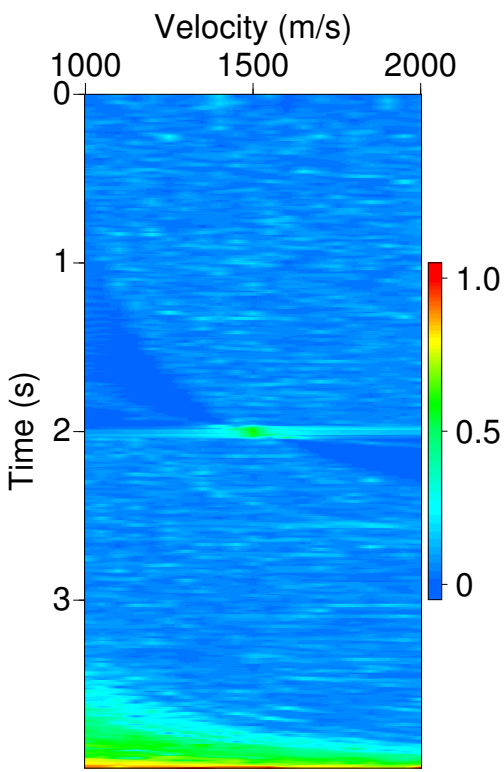

(c)

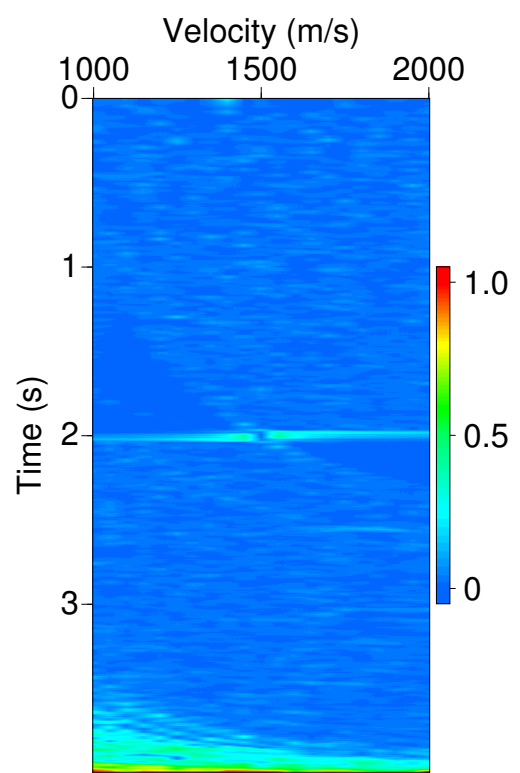

(b)

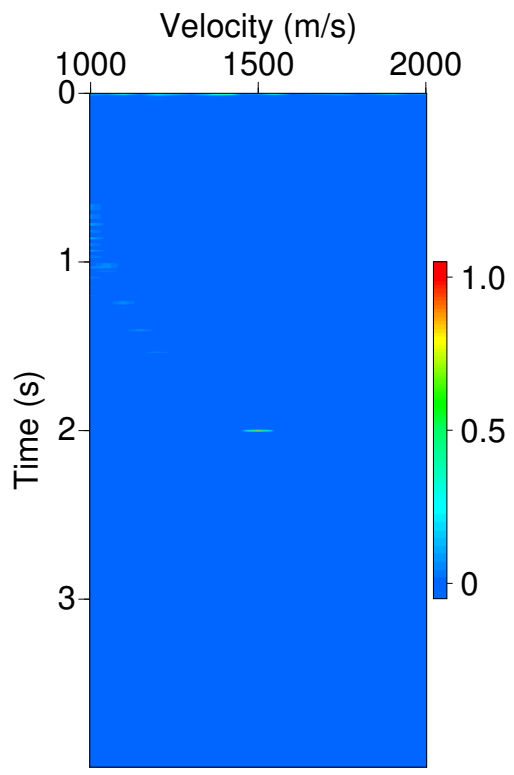

(d)

Figure 7 - Synthetic CMP gather in (a) with $80 \%$ of added random noise and its velocity spectra using conventional semblance in (b), AB semblance in (c), and Weighted AB semblance in (d).

of the moving window, used in the computation of all spectra, is $44 \mathrm{~ms}$ long. It is important to emphasize that $A B$ semblance presented a poorer resolution than conventional semblance, as we expected. There is no advantage in using the $A B$ semblance in data free from AVO effect. After using VFSA with 60 temperatures and 40 models, we obtained $a=3.2, b=1.8, c=4.0$ and 


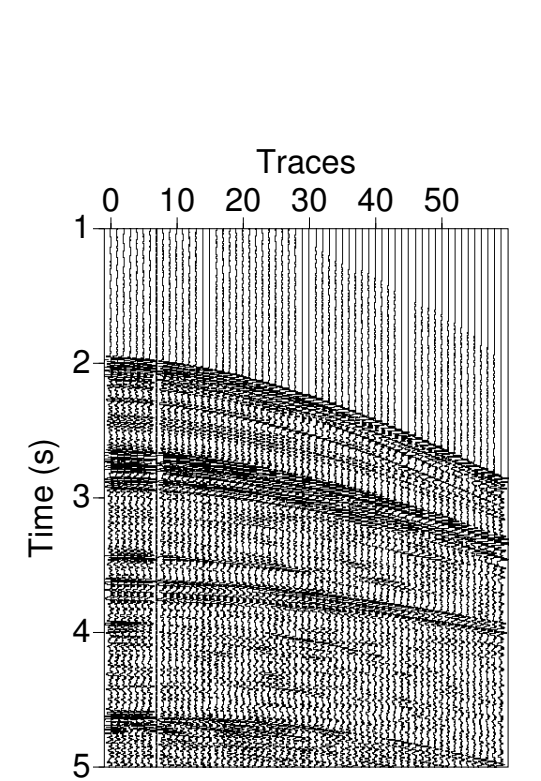

(a)

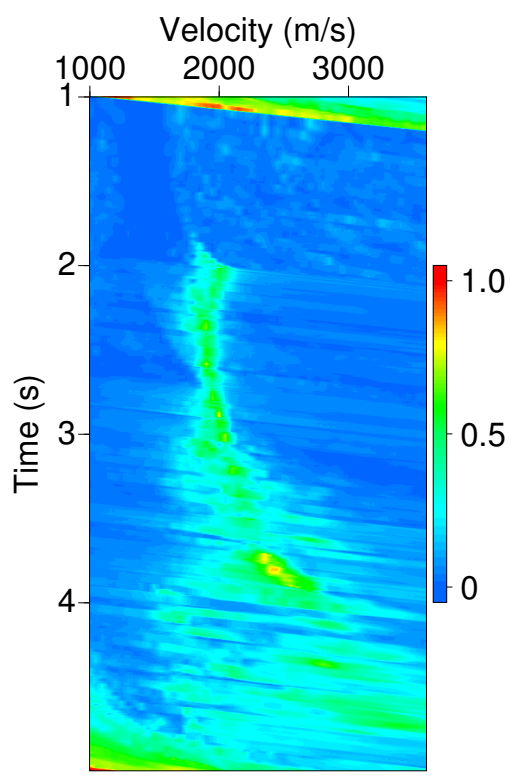

(c)

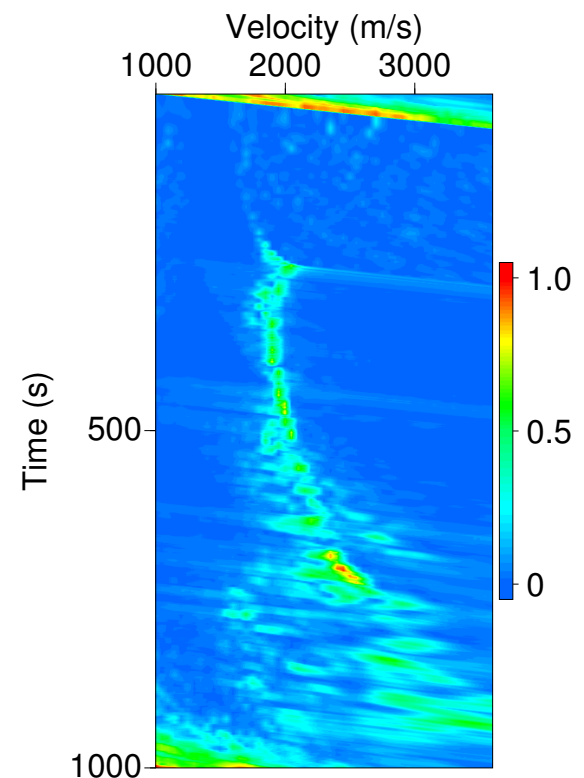

(b)

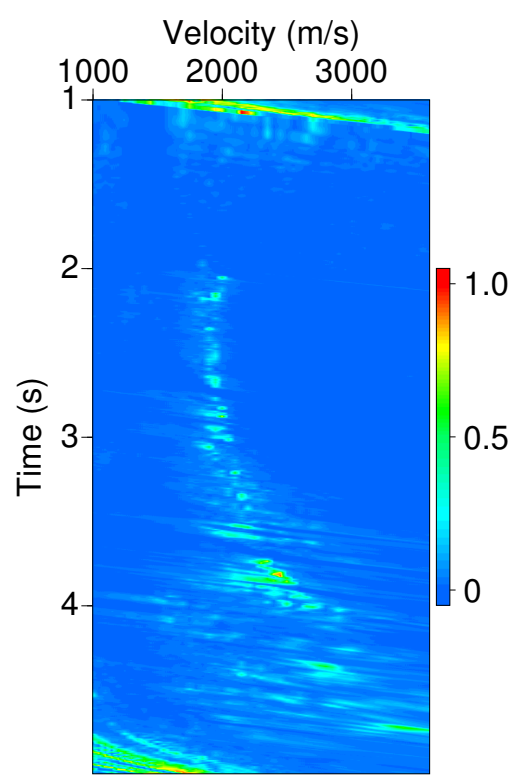

(d)

Figure 8 - Real CMP gather from Jequitinhonha Basin in (a) and its velocity spectra using conventional semblance in (b), AB semblance in (c), and Weighted AB semblance in (d). 


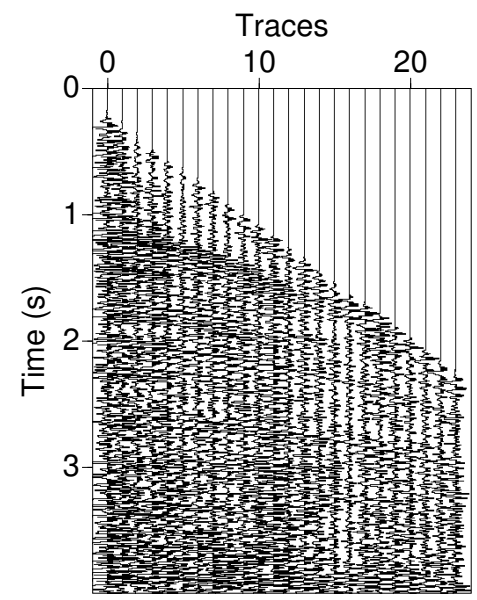

(a)

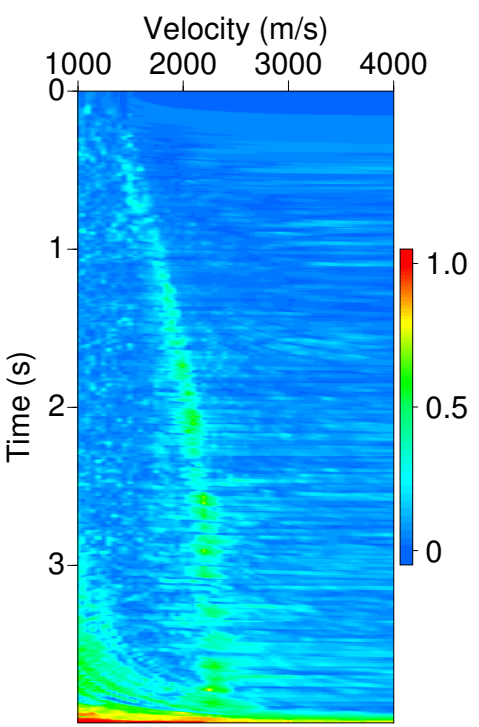

(c)

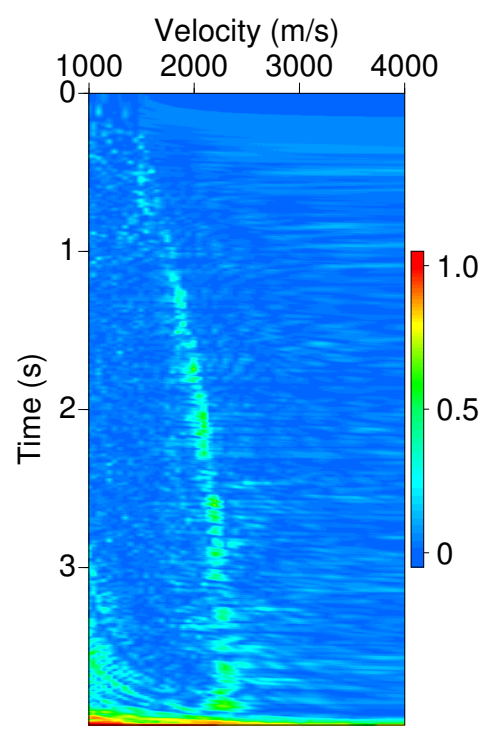

(b)

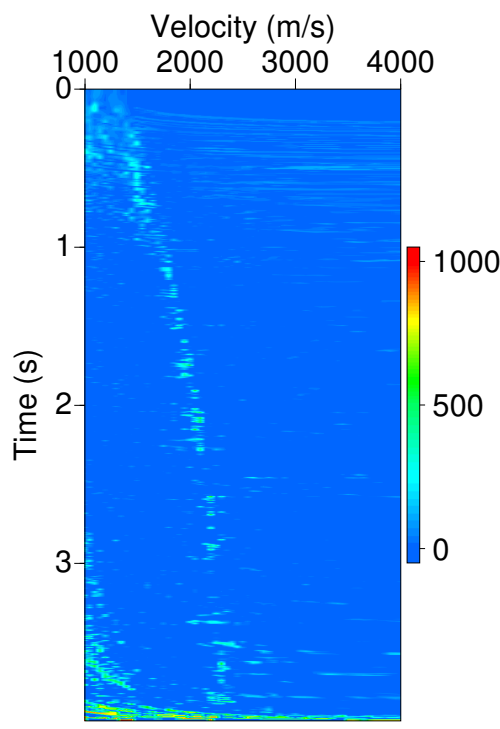

(d)

Figure 9 - Real CMP gather from Gulf of Mexico in (a) and its velocity spectra using conventional semblance in (b), AB semblance in (c), and Weighted AB semblance in (d).

$d=1.5$. Analyzing the data, we can realize that VFSA allowed estimates of the correct coefficient values, resulting in a velocity spectrum with the best resolution as compared with semblance and $A B$ semblance.

We also tested the method in a real CMP gather from Gulf of Mexico. The data parameters are 1000 time samples, 24 traces, and sampling interval of 4 milliseconds. In this CMP gather, we can see an AVO anomaly from $t=0.7$ to $1.1 \mathrm{~s}$. Ebrahimi et al. (2017a) used the same CMP gather. After using VFSA, we obtained the coefficients values $a=11.4, b=2.0, c=17.0$ and $d=3.5$. Figure 9 shows the CMP gather in (Fig. 9a), and its respective velocity spectra, normalized between zero and one 
in Figure 9b to Figure 9d, respectively. Comparing the results, we can conclude that conventional semblance does not detect the presence of events in the area of AVO anomaly. On the other hand, $A B$ semblance detects these events, but its resolution is poorer than conventional semblance. Weighted $A B$ semblance deals with the AVO phenomenon and improves considerably the resolution of the $A B$ semblance, this means that the VFSA provided the correct coefficient values, generating a velocity spectrum with an excellent resolution, making it easier the determination of the stacking velocities.

\section{CONCLUSIONS}

In this work, we applied the Weighted AB semblance method with a new approach: the use of the VFSA algorithm in the estimate of the coefficients of the weighting functions. Numerical experiments in both synthetic and real data presented satisfactory results, showing the effectiveness of the proposed method.

Conventional semblance is a robust coherence measure, but it cannot deal with the AVO effects. On the other hand, Weighted AB semblance showed to be an excellent method to identify reflection events by means of the velocity spectrum, mainly in data with AVO anomalies of the class 1 and 2 . The numerical experiments also showed that this measure presents a considerably better resolution than conventional semblance and $A B$ semblance, even in very noisy data.

The effectiveness in the application of the weighting functions to the coherence measure $A B$ semblance is directly related to the values of the coefficients that determine the graphical behavior of the functions. Since it is difficult to interpret the ECM matrix and consequently to obtain the correct coefficient values, the Very Fast Simulated Annealing inversion algorithm proved to be a good alternative to obtain the coefficient values with good effectiveness.

\section{ACKNOWLEDGMENTS}

This study was financed in part by the Coordenação de Aperfeiçoamento de Pessoal de Nível Superior - Brazil (CAPES) - Finance Code 001. The authors thank Fundação de Amparo à Pesquisa do Estado da Bahia - Brazil (FAPESB) for the financial support in Project PIE00005/2016 of the FAPESB Infrastructure Notice 003/2015.

\section{REFERENCES}

ABBAD B \& URSIN B. 2012. High-resolution bootstrapped differential semblance. Geophysics, 77(3): U39-U47.

BARROS TTL. 2012. Implementation Aspects of Eigendecomposition-Based High-Resolution Velocity Spectra. Master dissertation. Universidade Estadual de Campinas. São Paulo, Brazil. $72 \mathrm{pp}$.

BIONDI BL \& KOSTOV C. 1989. High-resolution velocity spectra using eigenstructure methods. Geophysics, 54(7): 832-842.

CHEN Y, LIU T \& CHEN X. 2015. Velocity analysis using similarity-weighted semblance. Geophysics, 80(4): A75-A82.

EBRAHIMI S, KAHOO A, CHEN Y \& PORSANI MJ. 2017 a. $A$ high-resolution weighted $A B$ semblance for dealing with amplitude-variation-with-offset phenomenon. Geophysics, 82(2): 85-93.

EBRAHIMI S, KAHOO A, PORSANI MJ \& KALATEH AN. 2017b. Obtaining high-resolution velocity spectra using weighted semblance. Exploration Geophysics, 48(3): 210-218.

FOMEL S. 2009. Velocity analysis using AB semblance. Geophysical Prospecting, 57(3): 311-321.

INGBER L. 1989. Very fast simulated re-annealing. Mathematical and Computer Modelling, 12(8): 967-973.

KEYS RG. 1989. Polarity reversals in reflections from layered media. Geophysics, 54(7): 900-905.

LUO S \& HALE D. 2012. Velocity analysis using weighted semblance. Geophysics, 77(2): U15-U22.

METROPOLIS N, ROSENBLUTH AW, ROSENBLUTH MN, TELLER AH \& TELLER E. 1953. Equation of state calculations by fast computing machines. The Journal of Chemical Physics, 21(6): 1087-1092.

OSTRANDER W. 1984. Plane-wave reflection coefficients for gas sands at nonnormal angles of incidence. Geophysics, 49(10): 1637-1648.

RUTHERFORD SR \& WILLIAMS RH. 1989. Amplitude-versus-offset variations in gas sands. Geophysics, 54(6): 680-688.

SACCHI MD. 1998. A bootstrap procedure for high-resolution velocity analysis. Geophysics, 63(5): 1716-1725.

SANTOS RS \& PORSANI MJ. 2017. Hybrid inversion of interval velocities in multiscale approach. Revista Brasileira de Geofísica, 35(4): 237-245.

SARKAR D, BAUMEL RT \& LARNER KL. 2002. Velocity analysis in the presence of amplitude variation. Geophysics, 67(5): 1664-1672.

SARKAR D, CASTAGNA J \& LAMB W. 2001. AV0 and velocity analysis. Geophysics, 66(4): 1284-1293.

SEN M \& STOFFA PL. 1995. Global optimization methods in geophysical inversion. Cambridge: Cambridge Un. Press. 302 pp. 
SOARES DL. 2013. Inversão de dados gravimétricos 2-D pelos métodos de Levenberg-Marquardt, resfriamento simulado muito rápido e híbrido. Master dissertation. Universidade Federal da Bahia. Salvador, BA, Brazil. $165 \mathrm{pp}$.

TANER MT \& KOEHLER F. 1969. Velocity spectra-digital computer derivation and applications of velocity functions. Geophysics, 34(6):
859-881. doi: 10.1190/1.1440058.

URSIN B, SILVA MG \& PORSANI MJ. 2014. Signal and traveltime parameter estimation using singular value decomposition. Geophysics, 79(5): U1-U14.

YAN J \& TSVANKIN I. 2008. AVO-sensitive semblance analysis for wide-azimuth data. Geophysics, 73(2): U1-U11.

Recebido em 30 de julho de 2019 / Aceito em 15 de novembro de 2019

Received on July 30, 2019 / Accepted on November 15, 2019 